\title{
Knowledge of Pregnant Women on Mother-to-Child Transmission of HIV in Meket District, Northeast Ethiopia
}

\author{
Tesfaye Birhane, ${ }^{1}$ Gizachew Assefa Tessema, ${ }^{2}$ \\ Kefyalew Addis Alene, ${ }^{3}$ and Abel Fekadu Dadi ${ }^{3}$ \\ ${ }^{1}$ Meket District Health Office, Meket, Ethiopia \\ ${ }^{2}$ Department of Reproductive Health, Institute of Public Health, University of Gondar, P.O. Box 196, Gondar, Ethiopia \\ ${ }^{3}$ Department of Epidemiology and Biostatistics, Institute of Public Health, University of Gondar, P.O. Box 196, Gondar, Ethiopia \\ Correspondence should be addressed to Gizachew Assefa Tessema; agizachew@gmail.com
}

Received 16 October 2014; Accepted 5 January 2015

Academic Editor: Sinuhe Hahn

Copyright (C) 2015 Tesfaye Birhane et al. This is an open access article distributed under the Creative Commons Attribution License, which permits unrestricted use, distribution, and reproduction in any medium, provided the original work is properly cited.

\begin{abstract}
Knowledge of pregnant women on the three periods of mother-to-child transmission (MTCT) of HIV has implication for child HIV acquisition. This study aims to assess the knowledge of pregnant women on mother-to-child transmission of HIV and to identify associated factors in Meket district, northeast Ethiopia. Logistic regression models were fitted to identify associated factors. Adjusted odds ratios (AOR) with 95\% confidence intervals (CI) were used to determine the presence and strength of association. About onefifth (19\%) of women were knowledgeable on mother-to-child transmission of HIV (95\% CI: 15.5\%, 22.4\%). Being urban resident (AOR: 2.69, 95\% CI: 1.48, 4.87), having primary education (AOR: 2.41, 95\% CI: 1.03, 5.60), reporting receiving information on HIV from health care providers (AOR: 3.24, 95\% CI: 1.53, 6.83), having discussion with partner about mother-to-child transmission of HIV (AOR: 2.64, 95\% CI: 1.59, 4.39), and attending antenatal care (AOR: 5.80, 95\% CI: 2.63, 12.77) were positively associated with increased maternal knowledge of mother-to-child transmission of HIV. Knowledge of mother-to-child transmission of HIV among pregnant women was low. Providing information, especially for rural women and their partners, is highly recommended.
\end{abstract}

\section{Background}

Vertical transmission of Human Immunodeficiency Virus (HIV) is still a major challenge in the world, especially in developing countries [1]. A report in 2012 reported about 35.3 million people are living with HIV of which 2.3 million are new infections whereas an estimated 3.3 million infected people are less than 15 years of age. Worldwide, there are about 6,300 new infections and 700 HIV-related deaths daily in 2012. Sub-Saharan Africa remains the region most heavily affected by HIV [2].

Without any intervention, the risk of a baby getting HIV infection from an infected mother ranges from 15\% to $25 \%$ in the developed nations and from $25 \%$ to $35 \%$ in developing countries. HIV transmission rate and timing are estimated to be $5 \%$ to $10 \%$ during pregnancy, $10 \%$ to $15 \%$ during delivery and $5 \%$ to $20 \%$ through breast-feeding. In general mother to child transmission contributes $15-45 \%$ of HIV acquisition for children [3].
The national adult HIV prevalence in Ethiopia is 1.2\% [4]. The national accelerated emergency plan includes three targeted objectives, that is, reaching $90 \%$ of pregnant women with access to antenatal care services, ensuring that all pregnant women have access to delivery by a skilled attendant, and providing antiretroviral prophylaxis to at least $80 \%$ of HIVpositive pregnant women [5].

It is estimated that 138,906 children less than 15 years are living with HIV in 2014. There are an estimated 3,886 new infections each year due to mother-to-child transmission [4]. However, timely interventions can reduce mother-to-child transmission to $2-5 \%[3,6,7]$. A global target has also been established to be achieved by the year 2015, that is, elimination of new HIV infections among children and prolonging the lives of the mothers with HIV [1].

According to Ethiopian Demographic and Health Survey (EDHS) report, about three-quarters of reproductive aged women know that HIV can be transmitted to a baby through breastfeeding [8]. 
The prevention of Mother-To-Child-Transmission (MTCT) of HIV is dependent on the knowledge of the mothers of the timing of possible transmission periods. However, knowledge of women on transmission periods of HIV from mother to child varies from country to country and has not been measured in Ethiopia at community level.

Different studies reported that sociodemographic factors like age [9], urban living [10], higher educational level [11], and being house wife [12] as factors that affect mothers' knowledge of MTCT of HIV. Studies conducted in southern and northwest Ethiopia $[10,12,13]$ reported that gravidity, parity, antenatal care (ANC) visits, and male partner discussion are factors associated with good knowledge of mothers on MTCT of HIV.

Maternal knowledge on MTCT is a corner stone of effective implementation of the World Health Organization (WHO) recommendation of the four-pronged approach to reduce mother-to-child transmission of HIV [1].

Despite the large challenge of vertical transmission of HIV, there were also limited community-based studies on women knowledge on mother-to-child transmission of HIV. Hence, this study attempts to fill the gap through assessing the level of knowledge of MTCT of HIV and its associated factors at Meket district, Northeast Ethiopia.

\section{Methods}

2.1. Study Design, Population, and Setting. A communitybased cross-sectional study design was conducted in Meket district, northeast Ethiopia, from March 8 to 21, 2014. Meket district is located $665 \mathrm{~km}$ north of Addis Ababa, the Ethiopian capital city. The district has an estimated population size of 254,520 of which 59,939 are reproductive aged women, and an estimated 8,246 were pregnant women. Those pregnant women are living in Meket district were constituted our study population.

2.2. Sample Size and Sampling Procedure. Sample size was determined using single population proportion formula with the assumptions of $95 \%$ level of confidence, $12 \%$ proportion of knowledgeable women on MTCT of HIV [12], 4\% of margin of error, and design effect of two. Finally, considering a nonresponse rate of $10 \%$, the total sample size was calculated to be 556. Multistage stratified sampling technique was used to select the study participants. In the district, there are two urban and 46 rural kebeles. Hence, in the first step, eight rural kebeles were randomly selected; however, since they are few, all the urban kebeles were included. On the second stage, 79 pregnant women from urban kebeles and 477 pregnant women from rural kebeles were randomly selected.

2.3. Operational Definitions. In the present study, pregnant woman was regarded as being knowledgeable on MTCT if she correctly identified the three different modes/periods of MTCT of HIV; otherwise she was classified as nonknowledgeable. Comprehensive knowledge of HIV was also measured if a pregnant woman correctly identified three modes of transmission of HIV (unsafe sexual practice, blood transfusion, and MTCT) and recognized two common misconceptions.

Comprehensive knowledge about HIV/AIDS was measured after posing the following questions: (1) knowing that condom use and limiting sex partners to one uninfected partner are HIV prevention methods, (2) being aware that a healthy-looking person can have HIV, and (3) rejecting the two most common local misconceptions, that is, HIV/AIDS can be transmitted through mosquito bites and by supernatural means in Ethiopia [8].

2.4. Data Collection Procedures. Data were collected using pretested, structured, and interviewer administered questionnaire. The questionnaire was prepared after reviewing relevant literatures. Five female nurses supervised by two BSc health professionals collected the data. For eligible women who were not at home during our first attempt, the interviewers revisited the participant's home at least two times before excluding the participant.

Training was given to the data collectors about informed consent, techniques of interviewing, data collection procedures, and different sections of the questionnaire. Supervisors and principal investigators checked the questionnaire on its completeness and consistency on the daily basis.

2.5. Data Processing and Analysis. The data were entered into EPI info version 3.5.3 statistical software and then sorted, cleaned, and analyzed by using SPSS version 20 statistical package. Descriptive statistics were done to describe the study participants in relation to relevant variables. Both bivariate and multiple logistic regression analyses were carried out to see the effect of sociodemographic factors, maternal condition factors, and other factors on the knowledge of MTCT of HIV and to control cofounding. Odds ratios with 95\% CI were computed to identify factors associated with mothers' MTCT knowledge.

2.6. Ethical Consideration. Ethical clearance was obtained from the Research and Ethical Review Committee (REC) at the Institute of Public Health, College of Medicine and Health Science of University of Gondar. Permission letter was secured from Meket District Health Office. Written informed consent was taken from each study participant after reading the consent form. The purpose and benefit of the study and their right to withdraw at any time were also delivered to each participant prior to the interview. Confidentiality of the information was maintained throughout by using anonymity identifiers, keeping their privacy by interviewing them individually.

\section{Results}

3.1. Sociodemographic Characteristics of Pregnant Women. Five hundred forty-two pregnant women participated in the study (97.5\% response rate). The majority (85.4\%) were rural dwellers. The mean age of the study participants was 29.45 years $(\mathrm{SD}=5.4)$. Four hundred and sixty $(84.9 \%)$ were married, $196(36.2 \%)$ were able to read and write, and nearly four-fifths (80.1\%) were homemaker (Table 1). 
TABLE 1: Selected sociodemographic characteristics of respondents, Meket district, northeast Ethiopia, $2014(n=542)$.

\begin{tabular}{|c|c|c|}
\hline Variables & Frequency & Percent \\
\hline \multicolumn{3}{|l|}{ Age (years) } \\
\hline $15-24$ & 99 & 18.3 \\
\hline $25-34$ & 326 & 60.1 \\
\hline $35-49$ & 117 & 21.6 \\
\hline \multicolumn{3}{|l|}{ Residence } \\
\hline Urban & 79 & 14.6 \\
\hline Rural & 463 & 85.4 \\
\hline \multicolumn{3}{|l|}{ Marital status } \\
\hline Married & 460 & 84.9 \\
\hline Single & 27 & 5 \\
\hline Divorced & 55 & 10.1 \\
\hline \multicolumn{3}{|l|}{ Educational status } \\
\hline Unable to read and write & 176 & 32.5 \\
\hline Able to read and write only & 196 & 36.2 \\
\hline Primary & 127 & 23.4 \\
\hline Secondary and above & 43 & 7.9 \\
\hline \multicolumn{3}{|l|}{ Occupation } \\
\hline Housewife & 434 & 80.1 \\
\hline Student & 26 & 4.8 \\
\hline Merchant & 55 & 10.1 \\
\hline Government employee & 27 & 5.0 \\
\hline \multicolumn{3}{|l|}{ Income (ETB) } \\
\hline$\leq 450$ & 458 & 84.5 \\
\hline $451-999$ & 77 & 14.2 \\
\hline$\geq 1000$ & 7 & 1.3 \\
\hline
\end{tabular}

3.2. Reproductive Health Related Characteristics and MTCT of HIV Knowledge. One hundred sixty-one (19.7\%) were pregnant for the first time. More than half (57.6\%) had ANC during their current pregnancy. Nearly two-thirds (63.8\%) had received information about HIV/AIDS from health care providers.

Half $(51.8 \%)$ of the respondents received information about HIV, antenatal care (65.7\%), mother-to-child transmission of HIV (40.6\%), and infant feeding with their partners (21.4\%) (Table 2).

3.3. Knowledge of Pregnant Women on MTCT. One hundred three (19\%) (95\% CI: $15.5 \%, 22.4 \%)$ were knowledgeable on MTCT of HIV. Most (84.5\%) heard about mother to child transmission of HIV. Among those who heard MTCT, more than two-thirds (70.7\%) mentioned labor/delivery as a time of HIV transition from mother to child. 225 (41.5\%) pregnant women identified at least two periods of motherto-child transmission of HIV. Nearly two-thirds (63.8\%) had comprehensive knowledge on HIV/AIDS, and another equivalent proportion of women heard about PITC (Table 2).

\subsection{Factors Associated with Knowledge of Pregnant Women} on MTCT of HIV. In multivariable analysis, higher levels of maternal education status, having received information about HIV from health professionals, and reported discussion of
TABLE 2: Reproductive health related characteristics and information received from health care providers, Meket district, northeast Ethiopia, $2014(n=542)$.

\begin{tabular}{lcc}
\hline Variables & Frequency & Percent \\
\hline Number of pregnancies & & \\
1 & 161 & 29.7 \\
$2-3$ & 276 & 50.9 \\
$\quad 4+$ & 105 & 19.4 \\
Gestational age & & \\
$\quad \leq 16$ & 10 & 1.9 \\
$17-24$ & 134 & 24.7 \\
$25-35$ & 333 & 61.4 \\
$\geq 36$ & 65 & 12.0 \\
Antenatal visit & & \\
$\quad$ Yes & 312 & 57.6 \\
$\quad$ No & 230 & 42.4 \\
Number of ANC visit $(n=312)$ & & \\
1 & 122 & 39.1 \\
$2-3$ & 176 & 56.4 \\
$4+$ & 14 & 4.5
\end{tabular}

Received information from health care providers

On HIV

Yes

No

On antenatal care

$\begin{array}{lll}\text { Yes } & 304 & 56.1\end{array}$

No

On MTCT

Yes 284

52.4

No

On infant feeding

Yes

181

33.4

No

361

66.6

Comprehensive knowledge of HIV/AIDS

Yes

346

63.8

No

36.2

Heard of PITC

Yes

63.7

No

345

36.3

Heard about MTCT

Yes

84.5

No

Know the means of transmission on MTCT $(n=458)$

During pregnancy 309

During labor/delivery

During breast feeding

Exact timing of MTCT answered by women

\begin{tabular}{lcc} 
None & 84 & 15.5 \\
One & 130 & 24.0 \\
Two & 225 & 41.5 \\
Three & 103 & 19.0 \\
\hline
\end{tabular}


TABLE 3: Crude and adjusted odds ratios (OR) and 95\% confidence intervals (CI) of factors associated with knowledge of mothers on MTCT of HIV among pregnant women, Meket district, $2014(n=542)$.

\begin{tabular}{|c|c|c|c|c|}
\hline \multirow{2}{*}{ Variables } & \multicolumn{2}{|c|}{ Knowledge on MTCT } & \multirow{2}{*}{ COR $(95 \%$ CI $)$} & \multirow{2}{*}{$\operatorname{AOR}(95 \% \mathrm{CI})$} \\
\hline & Yes & No & & \\
\hline \multicolumn{5}{|l|}{ Residence } \\
\hline Urban & 31 & 48 & $3.51(2.01,5.88)$ & $2.69(1.48,4.87)^{* *}$ \\
\hline Rural & 72 & 391 & 1.0 & 1.0 \\
\hline \multicolumn{5}{|l|}{ Age } \\
\hline $15-24$ & 34 & 65 & 1.0 & \\
\hline $25-34$ & 56 & 270 & $0.40(0.23,0.65)$ & \\
\hline $35-49$ & 13 & 104 & $0.24(0.11,0.48)$ & \\
\hline \multicolumn{5}{|l|}{ Education } \\
\hline Unable to read and write & 13 & 163 & 1.0 & 1.0 \\
\hline Able to read and write & 49 & 147 & $4.18(2.18,8.01)$ & $3.25(1.55,6.79)^{* *}$ \\
\hline Primary & 31 & 96 & $4.05(2.02,8.11)$ & $2.41(1.03,5.60)^{* *}$ \\
\hline Secondary and above & 10 & 33 & $3.80(1.53,9.39)$ & $2.05(0.71,5.88)$ \\
\hline \multicolumn{5}{|l|}{ ANC information from HP } \\
\hline Yes & 84 & 220 & $4.40(2.58,7.49)$ & \\
\hline No & 19 & 219 & 1.0 & \\
\hline \multicolumn{5}{|l|}{ HIV information from HP } \\
\hline Yes & 92 & 254 & $6.09(3.16,11.70)$ & $3.24(1.53,6.83)^{* *}$ \\
\hline No & 11 & 185 & 1.0 & 1.0 \\
\hline \multicolumn{5}{|c|}{ MTCT discussion with husband } \\
\hline Yes & 67 & 153 & $3.48(2.21,5.45)$ & $2.64(1.59,4.39)^{* *}$ \\
\hline No & 36 & 286 & 1.0 & 1.0 \\
\hline \multicolumn{5}{|l|}{ ANC discussion with husband } \\
\hline Yes & 95 & 261 & $8.10(3.84,17.08)$ & $5.80(2.63,12.77)^{* *}$ \\
\hline No & 8 & 178 & 1.0 & 1.0 \\
\hline
\end{tabular}

${ }^{* *}$ Statistically significant at $P$ value $<0.05$.

MTCT and ANC with their partners were positively associated with knowledge of mother-to-child transmission of HIV. Those women who live in the urban settings were about three more like to be knowledgeable than their rural counterparts (AOR: 2.69 , CI $(1.48,4.87))$. Those literate mothers were about three times more likely to be knowledgeable than who did not read and write (AOR: 3.25 , CI $(1.55,6.78)$ ). Likewise, a woman was 2.41 times more likely to be knowledgeable if she had completed primary school as compared to those who did not read and write (AOR: 2.41, CI $(1.04,5.60)$ ).

Pregnant women who received information on HIV from health care providers were about three times more likely to be knowledgeable than women who had not received information (AOR: 3.24 , CI $(1.54,6.83)$ ). Women who had discussions with their partner were more likely to be knowledgeable than those who had not (AOR: 5.80, CI $(2.63,12.78)$ ). Correspondingly, mothers who discussed MTCT with their partners were more likely to be knowledgeable than those who had not (AOR: 2.64, CI $(1.59,4.39)$ ) (Table 3).

\section{Discussion}

Being knowledgeable on MTCT of HIV and the fact that the risk of transmission can be reduced by using antiretroviral drugs are critical in reducing MTCT of HIV. This can contribute greatly towards the achievement of the Millennium Development Goals related to HIV.
This study revealed that $19 \%$ (95\% CI: $15.5 \%, 22.4 \%$ ) of respondents were knowledgeable on MTCT of HIV. This result is in line with a cross-sectional study conducted at Temeke District Hospital, Dar Es Salaam (15.7\%) [14]. However, it is higher than that of studies done in southern Ethiopia (11.5\%) and Gondar town $(8.5 \%)[10,13]$ but lower than a health institution based study in Debre Markos town, Ethiopia (42.3\%) [15]. This could be due to the difference in the study setting and accessibility of health facilities.

In the present study, nearly two-thirds of pregnant women had comprehensive knowledge on HIV/AIDS which is higher than studies in Yaoundé (23\%) [16], the Ethiopian Demographic and Health Survey (19\%) [8], and a study in Gondar town (59.8\%) [10].

Knowledge of pregnant women on MTCT of HIV among pregnant women was significantly varied based on their place of residence. Those pregnant women residing in urban areas were more likely to be knowledgeable when compared to the rural residents. This finding is in line with studies conducted at Gondar and Hawassa towns in Ethiopia [10, 13]. It might be due to the rural location and geographical inaccessibility and poor availability of nearby health services, compared with urban areas. This could also be partly explained due to the presence of media exposure amongst urbanites.

Educated pregnant women who were able to read and write were more likely to be knowledgeable than those who 
were unable to read and write. This supports the government attempt to address adult informal education. Pregnant women with primary education were also more likely to be knowledgeable than those who were unable to read and write. This result is in line with a previous study conducted in southern Ethiopia [12]. This could be because when the women become educated their access to information is also increased. With this regard, they might have access to print media exposure.

In this study, pregnant women who discussed and received information about HIV/AIDS from health care providers were more knowledgeable. They were found to be three times more likely to be knowledgeable than those who had not.

Spouse discussion on antenatal care follow-up was also positively associated with knowledge of MTCT. Those pregnant women who had discussions with their partners were six times more likely to be knowledgeable than those who had not discussed the issue. This is similar to reports from other studies $[12,17]$. This might be explained due to male partners possessing better knowledge on HIV transmission and eventually transfer this information to these pregnant women if discussion is triggered.

Pregnant women may receive information from a variety of sources about health services. Spouses having delivered information and participated in discussions about MTCT of HIV with their wives (40.6\%) were associated with good knowledge of the subject. Accordingly, pregnant women who had discussion with their partners were more than two times more likely to have good knowledge of MTCT. This might be because partner discussion in this regard could enhance their knowledge.

This study tried to assess pregnant women who did not attend health care facilities for ANC and HIV concerning their knowledge about MTCT of HIV. However, because of financial and time constraints, this study did not include the knowledge part of prevention of mother-to-child transmission of HIV.

\section{Conclusions}

Despite many efforts, the knowledge of pregnant women on mother-to-child transmission of HIV is low. If pregnant woman resides in urban environment, she attends school, if she receives information on HIV from health care providers, and if she attends antenatal care, she is more likely to be knowledgeable on MTCT of HIV. Strengthening women education and by reaching previously inaccessible parts of the community, integration of HIV, prevention of MTCT, and ANC service, is highly recommended. Moreover, strengthening discussion of MTCT with spouses is important.

\section{Conflict of Interests}

The authors declare that they have no competing interests.

\section{Authors' Contribution}

Tesfaye Birhane originated and wrote the proposal, participated in data collection, analyzed the data, and drafted the paper. Gizachew Assefa Tessema and Kefyalew Addis Alene approved the proposal with some revisions and participated in data analysis. Tesfaye Birhane, Gizachew Assefa Tessema, Kefyalew Addis Alene, and Abel Fekadu Dadi drafted the paper and sent for the journal for publication. All the authors read the paper.

\section{Acknowledgments}

The authors would like to acknowledge University of Gondar and Meket Health Office for technical and financial support, respectively. They are also grateful to the study participants for their time and data collectors for their commitment.

\section{References}

[1] UNAIDS, Global Plan Powards the Elimination of New HIV Infections among Children by 2015 and Keeping Their Mothers Alive, 2011-2015, Joint United Nations Programme on HIV/AIDS, Geneva, Switzerland, 2011.

[2] UNAIDS, Global Report: UNAIDS Report on the Global AIDS Epidemic 2013, United Nations Programme on HIV/AIDS, Geneva, Switzerland, 2013.

[3] World Health Organization, Mother to Child Transmission of $H I V$, World Health Organization, 2011.

[4] Federal HIV/AIDS Prevention and control Office, HIV/AIDS Estimates and Projections in Ethiopia, 2011-2016, Federal HIV/AIDS Prevention and control Office, Addis Ababa, Ethiopia, 2014.

[5] Ethiopia launches national plan to prevent new HIV infections among children, http://www.unaids.org/sites/default/files/en/ media/unaids/contentassets/documents/pressrelease/2011/12/ 20111204_PR_EthiopiaPMTCT_en.pdf.

[6] UNAIDS WHO, Technical Guidance Note for Global Fund HIV Proposals Prevention of Mother-to-Child Transmission of HIV, WHO/UNAIDS Global-Plan-Elimination-HIV-Children, 2011.

[7] Federal Ministry of Health, Manual for the Implementation of Prevention of Mother-to-Child Transmission of HIV in Ethiopia, Federal Ministry of Health, Addis Ababa, Ethiopia, 2011.

[8] CSA [Ethiopia] and ICF International, Ethiopian Demographic and Health Survey Report 2011, Central Statistical Agency, Addis Ababa, Ethiopia; ICF International, Calverton, Md, USA, 2012.

[9] O. E. Amoran, O. F. Salami, and F. A. Oluwole, "A comparative analysis of teenagers and older pregnant women in the utilization of prevention of mother to child transmission [PMTCT] services in, Western Nigeria," BMC International Health and Human Rights, vol. 12, article 13, 2012.

[10] M. T. Malaju and G. D. Alene, "Determinant factors of pregnant mothers' knowledge on mother to child transmission of HIV and its prevention in Gondar town, North West Ethiopia," BMC Pregnancy and Childbirth, vol. 12, article 73, 2012.

[11] Y. Luo and G.-P. He, "Pregnant women's awareness and knowledge of mother-to-child transmission of HIV in South Central China," Acta Obstetricia et Gynecologica Scandinavica, vol. 87, no. 8, pp. 831-836, 2008.

[12] A. Asefa and H. Beyene, "Awareness and knowledge on timing of mother-to-child transmission of HIV among antenatal care 
attending women in Southern Ethiopia: a cross sectional study," BMC Reproductive Health, vol. 10, no. 1, article 66, 2013.

[13] A. A. Abajobir and A. B. Zeleke, "Knowledge, attitude, practice and factors associated with prevention of mother-to-child transmission of HIV/AIDS among pregnant mothers attending antenatal clinic in Hawassa referral hospital, South Ethiopia," Journal of AIDS and Clinical Research, vol. 4, no. 6, 2013.

[14] N. Mujumali, Knowledge and attitude on prevention of mother to child transmission of hiv among pregnant women attending reproductive and child health clinic at Temeke district, Muhimbili University of Health and Allied Sciences, 2011.

[15] Z. Moges and A. Amberbir, "Factors associated with readiness to VCT service utilization among pregnant women attending antenatal clinics in Northwestern Ethiopia: a health belief model approach," Ethiopian Journal of Health Sciences, vol. 21, supplement 1, pp. 107-115, 2011.

[16] A.-C. Zoung-Kanyi Bissek, I. E. Yakana, F. Monebenimp et al., "Knowledge of pregnant women on mother-to-child transmission of HIV in Yaoundé," The Open AIDS Journal, vol. 5, no. 1, pp. 25-28, 2011.

[17] A. I. Olugbenga-Bello, E. A. Oladele, A. A. Adeomi, and A. Ajala, "Perception about HIV testing among women attending antenatal clinics at Primary Health Centres in Osogbo, Southwest, Nigeria," Journal of AIDS and HIV Research, vol. 4, no. 4, pp. 105-112, 2012. 


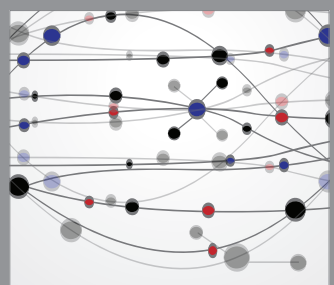

The Scientific World Journal
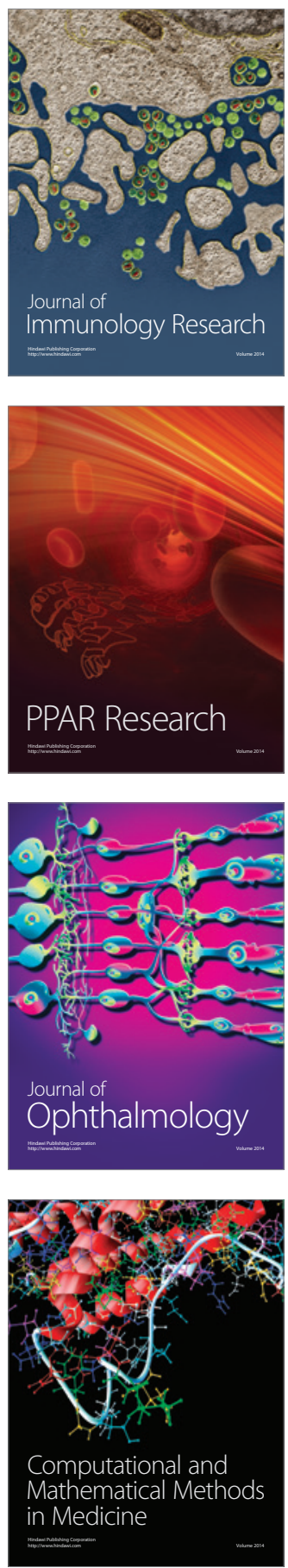

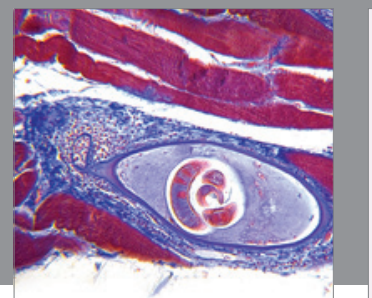

Gastroenterology

Research and Practice
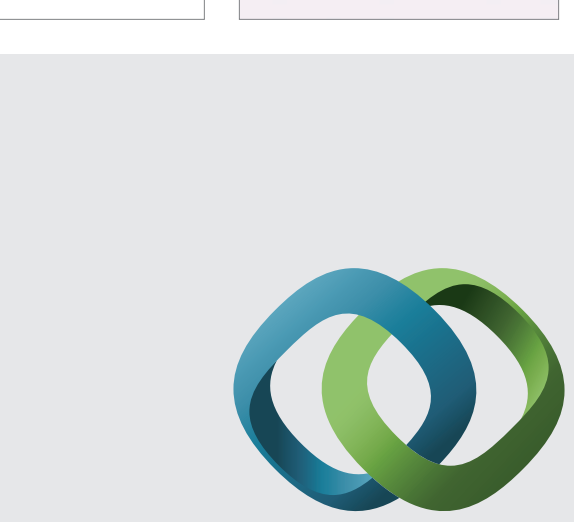

\section{Hindawi}

Submit your manuscripts at

http://www.hindawi.com
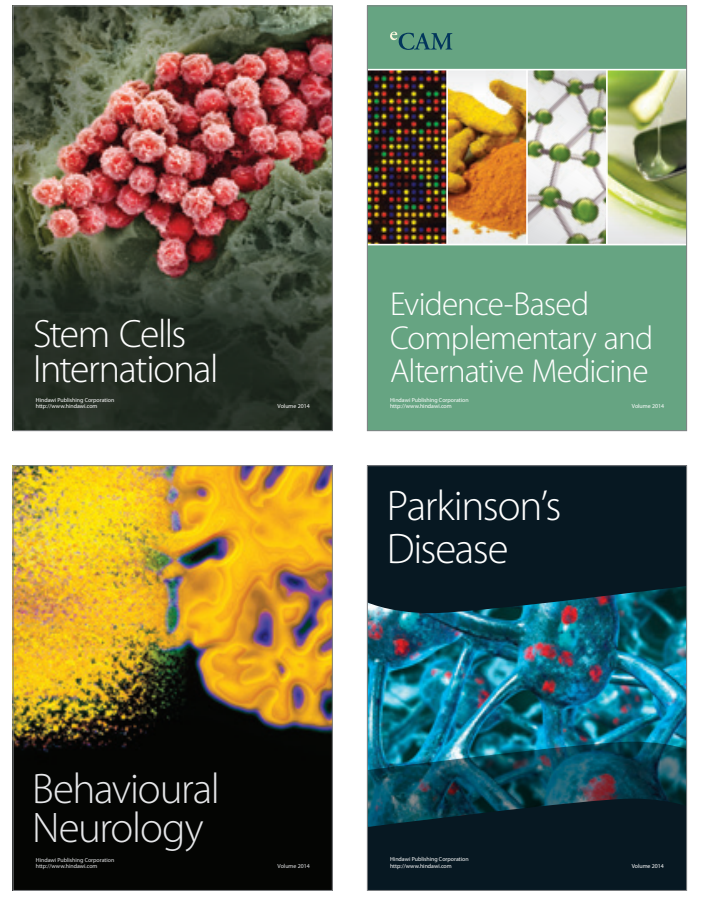
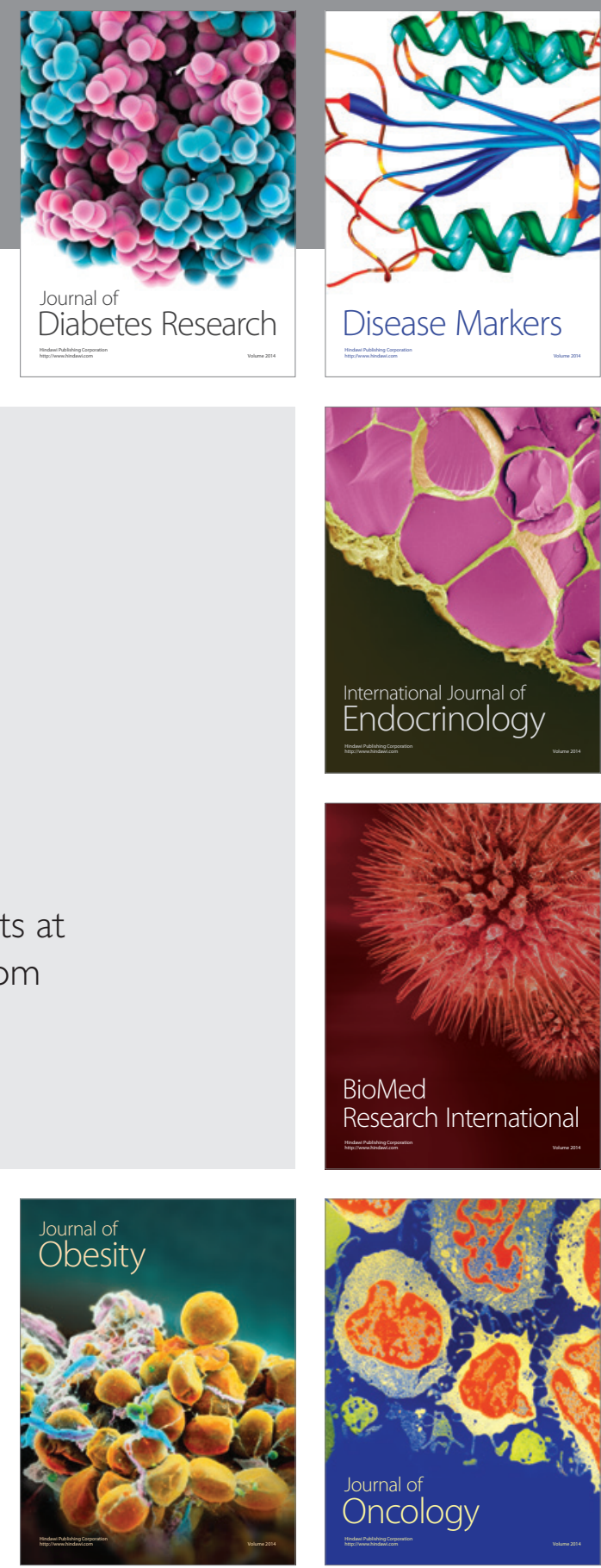

Disease Markers
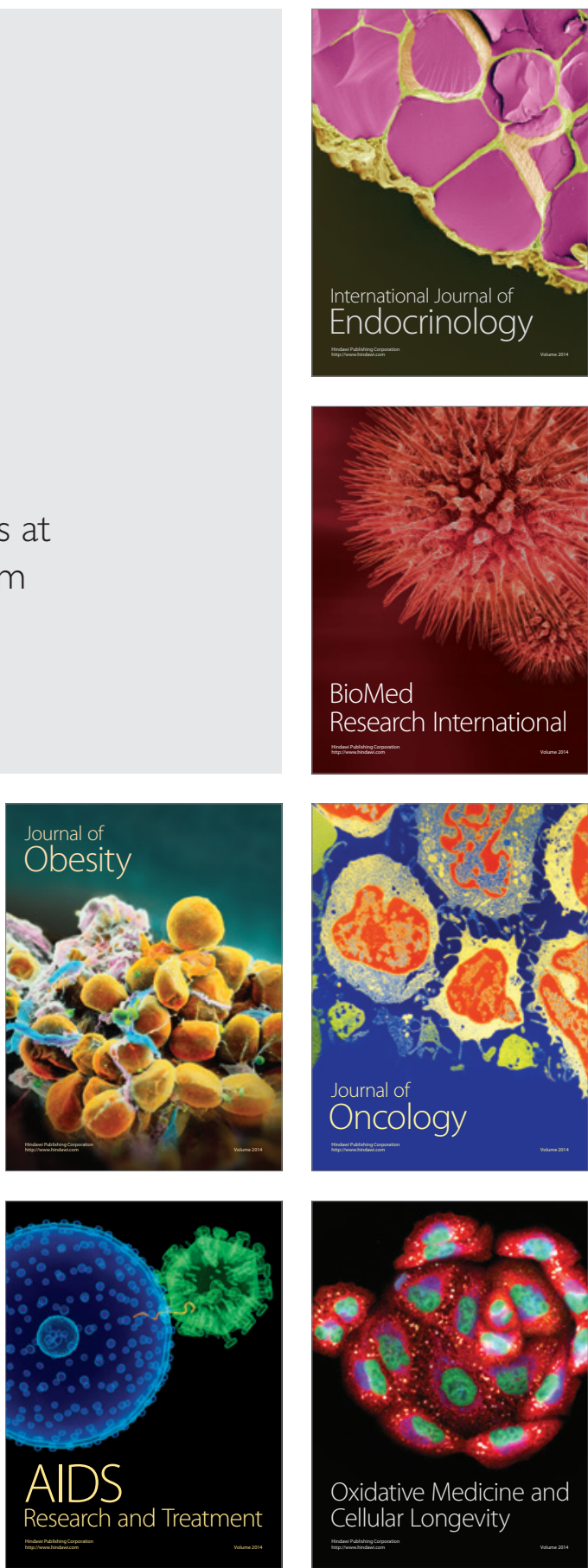